\title{
Does Kinesiophobia Mediate the Relationship between Pain Intensity and Disability in Individuals with Chronic Low-Back Pain and Obesity?
}

\author{
Giorgia Varallo ${ }^{1,2} \mathbb{D}^{\mathbb{D}}$, Federica Scarpina ${ }^{3,4}$, Emanuele Maria Giusti ${ }^{1,2, *} \mathbb{C}$, Roberto Cattivelli ${ }^{1,2} \mathbb{D}$, \\ Anna Guerrini Usubini ${ }^{1,2}$, Paolo Capodaglio ${ }^{5,6}{ }^{-}$and Gianluca Castelnuovo ${ }^{1,2}$ \\ 1 Istituto Auxologico Italiano IRCCS, Psychology Research Laboratory, San Giuseppe Hospital, \\ 28824 Verbania, Italy; g.varallo@auxologico.it (G.V.); r.cattivelli@auxologico.it (R.C.); \\ anna.guerriniusubini@unicatt.it (A.G.U.); gianluca.castelnuovo@auxologico.it (G.C.) \\ 2 Department of Psychology, Catholic University of Milan, 20123 Milan, Italy \\ 3 Rita Levi Montalcini Department of Neurosciences, University of Turin, 10124 Turin, Italy; \\ f.scarpina@auxologico.it \\ 4 Istituto Auxologico Italiano IRCCS, Unit of Neurology and Neurorehabilitation, San Giuseppe Hospital, \\ 28824 Verbania, Italy \\ 5 Istituto Auxologico Italiano IRCCS, Orthopaedic Rehabilitation Unit and Clinical Lab for Gait Analysis and \\ Posture, San Giuseppe Hospital, 28824 Verbania, Italy; p.capodaglio@auxologico.it \\ 6 Department of Surgical Sciences, Physical and Rehabilitation Medicine, University of Turin, 10121 Turin, Italy \\ * Correspondence: e.giusti@auxologico.it; Tel.: +39-0323-4338
}

\section{check for} updates

Citation: Varallo, G.; Scarpina, F.; Giusti, E.M.; Cattivelli, R.; Guerrini Usubini, A.; Capodaglio, P.; Castelnuovo, G. Does Kinesiophobia Mediate the Relationship between Pain Intensity and Disability in Individuals with Chronic Low-Back Pain and Obesity?Brain Sci. 2021, 11, 684. https://doi.org/10.3390/ brainsci11060684

Academic Editor: Vincenzo Donadio

Received: 3 May 2021

Accepted: 20 May 2021

Published: 22 May 2021

Publisher's Note: MDPI stays neutral with regard to jurisdictional claims in published maps and institutional affiliations.

Copyright: (c) 2021 by the authors. Licensee MDPI, Basel, Switzerland. This article is an open access article distributed under the terms and conditions of the Creative Commons Attribution (CC BY) license (https:/ / creativecommons.org/licenses/by/ $4.0 /)$.

\begin{abstract}
Individuals suffering from chronic low-back pain and obesity face severe physical and functional limitations. According to the fear-avoidance model, kinesiophobia might play a crucial role in the relationship between pain intensity and disability. Thus, the purpose of this study was to verify the role of kinesiophobia as a mediator in the association between pain intensity and disability in individuals with both chronic low-back pain and obesity. A total of 213 individuals with chronic low-back pain and obesity were included in the study. The level of kinesiophobia, pain intensity and disability were all assessed using self-reported questionnaires. We verified through a simple mediation analysis that kinesiophobia partially mediated the association between pain intensity and disability in our sample. According to our findings, we emphasize the crucial role of kinesiophobia as a psychological factor that should be addressed in chronic low-back pain rehabilitative protocols to reduce disability in individuals with obesity.
\end{abstract}

Keywords: chronic low-back pain; disability; kinesiophobia; fear of movement; fear-avoidance model; obesity; rehabilitation; clinical psychology

\section{Introduction}

Chronic low-back pain (CLBP) occurs when pain lasts for more than three months and is associated with emotional distress, decreased physical functioning, and is not better accounted for by another disease [1]. CLBP is one of the main causes of disability worldwide [2] and its prevalence is increasing, despite different available treatments, such as surgery and pharmacotherapy [3].

The prevalence of CLBP in the general population is directly associated with higher body mass index (BMI), and obesity (defined as BMI $\geq 30 \mathrm{~kg} / \mathrm{m}^{2}$ [4]) is a risk factor for its development $[5,6]$. Thus, as the obesity rates rise, so do the rates of musculoskeletal diseases and associated physical disabilities [7,8].

Individuals with CLBP and obesity experience significant functional limitations and disability $[9,10]$. They do, indeed, face a dual problem: movement difficulties caused by excess weight, as well as pain interference in daily life due to CLBP [11]. Both conditions have an impact on one another. Thus, reduced physical activity due to pain can result in weight gain $[12,13]$. 
The intensity of pain plays a role in determining the level of disability in acute lowback $[14,15]$ and chronic low-back pain sufferers [16]. However, the level of functional disability experienced by people with acute low-back and chronic low-back pain is not entirely explained by the pain intensity level [14-16].

Recognizing which factors intervene in the relationship between pain intensity and disability may have a significant impact on how clinicians plan rehabilitative interventions, especially which rehabilitation targets should be prioritized. Nonetheless, the pathways by which pain leads to disability in people with obesity and CLBP remain still unknown $[17,18]$.

According to the bio-psycho-social model, both pain intensity, and biomedical and psychological factors converge to cause functional disability. The fear-avoidance model (FAM) [19-21] has become increasingly important in this clinical field. This model underlines the role of cognitive-behavioral factors in the onset of chronic pain. Indeed, according to the FAM [19-21] pain intensity and disability are linked via psychological processes, such as pain catastrophizing and fear of pain associated with movements (i.e., kinesiophobia) $[19,20,22]$.

Kinesiophobia is an exaggerated, irrational fear of movement and activities, caused by the perception of vulnerability as a result of a painful injury or fear of reinjury [23]. This fear can lead chronic pain sufferers to avoid activities and movements that they associate with the occurrence or exacerbation of pain [21,24], resulting in deconditioning and disuse $[19,25]$.

In individuals with chronic pain, kinesiophobia is consistently associated with pain intensity [26] and disability [27]. According to emerging research, kinesiophobia is a factor associated with pain intensity $[28,29]$ and disability $[29,30]$ also in individuals with both obesity and CLBP. Moreover, they report suffering from higher levels of kinesiophobia, as well as higher perceived disability and greater mobility impairment than their normalweight counterparts [12]. Indeed, individuals with obesity frequently complain about dyspnea, musculoskeletal discomfort, and joint pain during physical activity [31], which may make them perceive the physical activity as even more unpleasant and may alter the individual perception of the benefits of movement and physical activity.

However, it appears that kinesiophobia is not only associated with pain intensity [26,29] and disability $[27,29]$ but is also an intervening variable that explains their relationship. Indeed, the FAM [19-21] suggests that pain intensity influences disability via the mechanism of kinesiophobia. Previous cross-sectional mediation studies on chronic low-back pain sufferers [16,32] and individuals with a whiplash injury [33] appear to confirm that kinesiophobia plays the role of mediator between pain intensity and disability.

Thus, our research aims to address the following question: is kinesiophobia a psychological factor that can explain how pain intensity causes disability in individuals with CLBP and obesity?

We conducted this cross-sectional study to address this question with the aim of verifying the mediating role of kinesiophobia. As suggested by previous findings relative to chronic back-pain sufferers [16,32] we hypothesized that in people with obesity and CLBP, kinesiophobia would partially mediate the association between pain intensity and disability.

\section{Materials and Methods}

The Ethical Committee of Istituto Auxologico Italiano (code 2020_02_18_04) approved this study. An informed consent document was read, understood, and signed by all participants. All procedures were carried out in line with the Helsinki Declaration of 1975, as revised in 1983.

\subsection{Participants}

A cross-sectional study was carried out. Participants were consecutively recruited, from 1 December 2019 to 28 February 2020, at the Istituto Auxologico Italiano IRCCS, 
U.O. di Riabilitazione Osteoarticolare, Ospedale S. Giuseppe, Piancavallo, Italia, at the beginning of a month-long hospitalization for weight loss and physical rehabilitation,

Inclusion criteria were as follows: age in years $>18$ and $\leq 65$; obesity, as measured by a body mass index (i.e., BMI computed as the weight in kilograms divided by the square of height in meters: $\mathrm{kg} / \mathrm{m}^{2}$ ) $\geq 30$ [4] and CLBP, defined as low back pain duration $>$ three months [1], diagnosed by a rheumatologist at the beginning of the hospitalization.

Exclusion criteria included physical or mental inability to provide signed informed consent; pain duration < three months; diagnosis of another disease that might explain lumbar pain; diagnosis of fracture, neoplasia, bone metastasis, stenosis, which might explain the low back pain; postoperative pain; neurogenic or radicular condition; neurological disease.

Demographic and clinical data were collected through a self-report form completed at the beginning of hospitalization. The completion of the psychological questionnaire was supervised by a researcher.

\subsection{Measures}

The level of disability was measured using the Italian version [34] of the RolandMorris Disability Questionnaire (RMDQ) [35]. The RMDQ consists of 24 dichotomous items covering daily activities and asks participants to rate their level of difficulty in performing them. A total score ranging from 0 to 24 is computed. Higher levels of painrelated disability are reflected by higher scores. The Italian version of the RMDQ showed levels of reliability and validity comparable to the original version [34]. In the current study, the internal consistency was good (Cronbach's $\alpha=0.82$ ).

The Numeric Pain Rating Scale (NPRS) [36] was used to evaluate pain intensity levels. The NPRS is composed of an 11-point scale (anchors $0=$ no pain, $10=$ worst possible pain). The NPRS is a well-validated and widely used measure in chronic pain conditions [37].

To assess the level of kinesiophobia, we used the Italian version [38] of the Tampa Scale of Kinesiophobia (TSK) [38]. The TSK consists of 13 items that range from "strongly agree" to "strongly disagree" on a 4-point Likert scale [39]. The TSK has two subscales relative to activity avoidance (i.e., belief that pain-inducing activities should be avoided) and harm (i.e., belief that pain is a sign of bodily harm). The total score ranges from 13 to 52; higher scores indicating higher levels of kinesiophobia [38]. The TSK has been validated in CLBP [23]. The Italian version of the TSK shows a good factorial structure and acceptable psychometric properties [38]. In the current sample, the internal consistency of this measure was excellent (Cronbach's $\alpha=0.90$ ).

\subsection{Statistical Analysis}

Descriptive statistics were calculated in terms of means, standard deviations, and ranges for continuous variables and frequencies and percentages for categorical variables.

Preliminarily, Pearson's correlation was used to verify the relationship between age, BMI, and scores at NPRS, TSK, and RMDQ; whereas the point-biserial correlation was used to study the association between age, BMI, scores at NPRS, TSK, RMDQ, and sex. Correlation coefficients were classified according to Cohen [40] ( $0.10=$ small; $0.30=$ medium; $0.50=$ large $)$. Variables with high correlation indicating multicollinearity issues $(\mathrm{r}>0.90)$, or variables that were not correlated with either pain intensity or disability, were excluded from the following mediation analyses.

A simple mediation analysis was carried out. Mediation analysis is a method of investigating an explanatory mechanism by determining the extent to which an intermediary variable (i.e., mediator) explains the association between a predictor and outcome [41].

In our model, kinesiophobia (M) was entered as a mediator in the association between pain intensity (i.e., predictor, X) and the level of disability (i.e., outcome, Y), see Figure 1. 
A

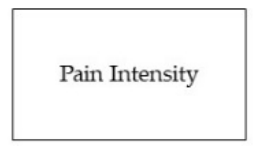

Total effect, c path

B

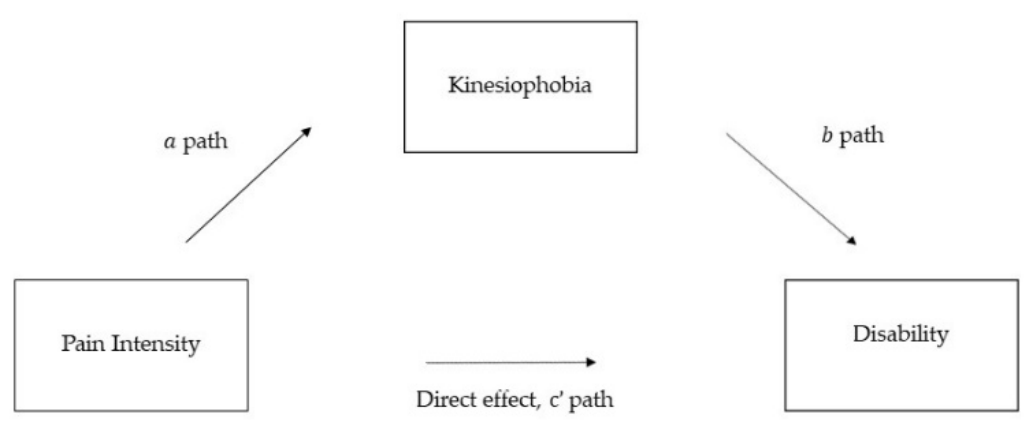

Figure 1. Conceptual diagram of the mediation model. Note. (A) The total effect is the effect of pain intensity on disability (c). (B) The direct effect ( $\left.\mathrm{c}^{\prime}\right)$ is the effect of pain on disability after controlling for the mediator variables. 'a path' is the association between pain intensity and kinesiophobia. 'b path' is the association between kinesiophobia and disability controlling for pain intensity.

According to Baron and Kenny [42], four conditions had to be met to confirm mediation: (i) pain intensity should be related to disability (total effect; $c$ path $=c^{\prime}+a \times b$ ); (ii) pain intensity should be related to kinesiophobia (a path); (iii) controlling for pain intensity, kinesiophobia should be significantly associated with disability (b path); (iv) the relationship between pain intensity and disability should be reduced (direct effect, $c^{\prime}$ path) when controlling for kinesiophobia (indirect effect, $a \times b$ ).

The simple mediation model was tested using Jamovi 1.2 [43]. We used bias-corrected bootstrap confidence intervals (CI-BC) for inference about indirect effects that it does not require the assumption of normality and reduce the type I error $[44,45]$. An estimate of the indirect effect was obtained from the mean of 5000 bootstrap samples and $95 \% \mathrm{CI}-\mathrm{BC}$. The indirect effect is considered statistically significant when confidence intervals (CI-BC) do not include zero.

According to Fritz and Mackinnon [46], our sample size is sufficient for a mediated effect including small-to-medium $(0.26) \mathrm{a}$ and $\mathrm{b}$ paths with a 0.80 power.

\section{Results}

\subsection{Participants' Characteristics}

Overall, two hundred and thirteen individuals were included in this study. Table 1 shows the demographic and clinical characteristics of participants; also, we report means, standard deviations, and ranges, for the three main measures (i.e., NPRS: Numeric Pain Rating Scale; TSK: Tampa Scale of Kinesiphobia; RMDQ: Roland Morris Disability Questionnaire).

Table 1. Demographic and clinical characteristics of the sample $(n=213)$.

\begin{tabular}{cccc}
\hline & $\boldsymbol{n ( \% )}$ & Mean \pm sd & $\begin{array}{c}\text { Range } \\
\text { (Min-Max) }\end{array}$ \\
\hline Sex & $\mathrm{M}=68(31.9) ; \mathrm{F}=145(68.1)$ & & \\
Age (in years) & & $56.8 \pm(9.93)$ & $26-65$ \\
BMI (Kg/m $\left.{ }^{2}\right)$ & $44.9 \pm(8.81)$ & $30-60$ \\
NPRS & $6.21 \pm(2.33)$ & $2-10$ \\
TSK & $30.4 \pm(7.72)$ & $13-50$ \\
RMDQ & $12.5 \pm(5.68)$ & $3-24$ \\
\hline
\end{tabular}

Note. M = Male; F = Female; BMI: Body Mass Index; NPRS: Numeric Pain Rating Scale; TSK: Tampa Scale of Kinesiphobia; RMDQ: Roland Morris Disability Questionnaire. 


\subsection{Preliminary Data Analysis}

There were no outliers or missing data. All correlation coefficients between NPRS, TSK, and RMDQ were less than 0.90 indicating the absence of multicollinearity [47]. According to the correlational analyses, age, sex, and BMI, were not significantly correlated with neither NPRS scores nor RMDQ scores (Table 2). Thus, these factors were not included in the mediation model [47].

Table 2. Pearson and point-biserial correlations coefficients between age, sex and BMI with the measures of pain intensity, kinesiophobia and disability.

\begin{tabular}{ccccccc}
\hline & Age (in Years) & Sex & BMI & NPRS & TSK & RMDQ \\
\hline Age (in years) & - & & & & & \\
Sex & 0.058 & - & & & & \\
BMI (Kg/m $\left.{ }^{2}\right)$ & 0.110 & -0.035 & - & & & \\
NPRS & 0.057 & -0.110 & -0.035 & - & & \\
TSK & 0.055 & 0.076 & 0.006 & $0.281^{* * *}$ & - & \\
RMDQ & 0.048 & -0.126 & 0.131 & $0.275^{* * *}$ & $0.373^{* * *}$ & - \\
\hline
\end{tabular}

Note. BMI: Body Mass Index; NPRS: Numeric Pain Rating Scale; TSK: Tampa Scale of Kinesiphobia; RMDQ: Roland Morris Disability Questionnaire. ${ }^{*} p<0.05,{ }^{* *} p<0.01,{ }^{* * *} p<0.001$.

\subsection{Mediation Analysis}

There was a significant total effect of the level of pain intensity on the level of disability ((i), total effect; c path), $(\mathrm{b}=0.669, \mathrm{SE}=0.157, p<0.001)$. Path a ((ii), association between pain intensity and kinesiophobia), $(\mathrm{b}=0.931, \mathrm{SE}=0.223, p<0.001,95 \%$ BC-CI: $0.499,1.360)$ and path $\mathrm{b}$ ((iii); association between kinesiophobia and disability), $(\mathrm{b}=0.236, \mathrm{SE}=0.049$, $p<0.001,95 \%$ BC-CI: $0.137,0.330)$ were significant. The indirect effect via kinesiophobia (iv; $\mathrm{a} \times \mathrm{b}$ path) was significant ( $\mathrm{b}=0.220, \mathrm{SE}=0.075, p=0.002,95 \%$ BC-CI: $0.094,0.377$ ). The direct effect ((iv); $\left.\mathrm{c}^{\prime}\right)$ was reduced compared to the total effect (c), but remained significant $(\mathrm{b}=0.449, \mathrm{SE}=0.161, p=0.005)$.

Overall, these findings revealed that the relationships between the level of pain intensity and the level of pain-related disability were partially mediated by the level of kinesiophobia. Results are reported in Table 3.

Table 3. Results of the simple mediation analysis.

\begin{tabular}{|c|c|c|c|c|c|c|}
\hline \multicolumn{7}{|c|}{ Path Estimates } \\
\hline & $\mathbf{b}$ & SE & LLCI & ULCI & $\mathbf{Z}$ & $p$-Value \\
\hline $\begin{array}{c}\text { Effect of pain intensity on } \\
\text { kinesiophobia (a path) }\end{array}$ & 0.931 & 0.223 & 0.499 & 1.360 & 4.17 & $<0.001^{* * *}$ \\
\hline $\begin{array}{l}\text { Effects of kinesiophobia on } \\
\text { disability (b path) }\end{array}$ & 0.236 & 0.049 & 0.137 & 0.330 & 4.77 & $<0.001^{* * *}$ \\
\hline $\begin{array}{l}\text { Effect of pain intensity on } \\
\text { disability }\left(c^{\prime}\right)\end{array}$ & 0.449 & 0.160 & 0.126 & 0.759 & 2.79 & $0.005^{* *}$ \\
\hline \multicolumn{7}{|c|}{ Mediation Estimates } \\
\hline & $\mathbf{b}$ & SE & LLCI & ULCI & $\mathbf{Z}$ & $p$-Value \\
\hline $\begin{array}{l}\text { Indirect effect of pain intensity on } \\
\text { disability through kinesiophobia } \\
\qquad(\mathrm{a} \times \mathrm{b} \text { path })\end{array}$ & 0.220 & 0.073 & 0.094 & 0.377 & 3.03 & $0.002 * *$ \\
\hline $\begin{array}{l}\text { Total effect of pain intensity on } \\
\text { disability through kinesiophobia } \\
\qquad\left(c^{\prime}+a \times b\right)\end{array}$ & 0.669 & 0.157 & 0.356 & 0.966 & 4.25 & $<0.001^{* * *}$ \\
\hline
\end{tabular}




\section{Discussion}

As previously reported [28-30], kinesiophobia is linked with both pain intensity [28,29] and disability $[29,30]$ CLPB sufferers with obesity. This study expanded on previous findings by verifying the role of kinesiophobia as a mediator. Specifically, we hypothesized that kinesiophobia partially mediates the association between pain intensity and disability.

Our findings appeared to support our hypothesis relative to a partial mediation of kinesiophobia in the association between pain intensity and disability. The results were consistent with previous cross-sectional studies $[16,32,48]$ that investigated this relationship in individuals with whiplash-associated disorders and CLBP, and they bring further evidence in favor of the FAM $[19,21]$.

Our findings broaden our understanding of the psychological factors that contribute to disability. As recently discussed, [49,50], focusing predominately on pain intensity might not be beneficial in the treatment of chronic pain. Indeed, pain might be considered a necessary but not sufficient condition for chronic disability, because not all individuals experiencing CLBP become chronically disabled. Our finding that kinesiophobia plays a role as a mediator in this relationship may help to clarify the circumstances under which disability develops. Experiencing pain can cause fear of injury and movement. resulting in avoidance and inactivity, perceived as protective behaviors, which in turn increases functional restrictions in daily life [20]. Aside from the role of pain intensity per se, the mediating effect of kinesiophobia suggested that how a person reacts to the pain experience (in this case with fear of movement and re-injury) may play a role in disability. Furthermore, kinesiophobia prevents the individual from confronting pain and exposing themselves to fear-inducing movement. Indeed, the FAM [20] hypothesizes confrontation, as opposed to avoidance, as a functional pain-coping strategy that leads to fear reduction in the individual. Taken together, our findings and previous research [14,51] indicate that kinesiophobia is a potential contributor to disability and might be one of the underlying mechanisms explaining how pain leads to disability.

Several clinical implications must be addressed. Developing more tailored treatments for CLBP requires a clarification of the mechanisms that lead to the onset, maintenance of disability. We suggested addressing kinesiophobia in therapeutic interventions especially in the case of associated obesity since individuals suffering from obesity and CLBP might not fully engage in treatments (e.g., physical therapy and exercise). During supervised rehabilitation, gradual exposure to movements that the patient associates with fear might be beneficial to reduce kinesiophobia [52]. A multidisciplinary team comprised of physicians, physical therapists, and psychologists might assist patients through the physical and psychological transition from living in a "pain-restricted" to a "pain-managed" state [30].

Some limitations should be addressed. Due to the cross-sectional design, causality could not be tested. Longitudinal studies should be done to overcome such a limitation. Additionally, since we recruited a care-seeking population recruited from a single center, our findings might not be generalizable to the overall population of individuals with obesity and CLBP. Because our findings support a partial mediation, the presence of other mediators that were not considered in this study should be considered and further examined. Moreover, unexpectedly no significant correlation was observed between pain intensity, disability, and BMI. Even though this result is not in line with some previous evidence [5,6], other studies found no relationship between BMI, pain intensity, and disability $[53,54]$. Research has suggested that BMI may be an oversimplified measurement of obesity $[9,55]$. For example, adiposity and the distribution of adipose tissue may be more useful in capturing the condition of obesity $[9,56,57]$.

Despite these limitations, this was the first study investigating the role of kinesiophobia in people with obesity and CBPL. Furthermore, this study was planned to comply with the quality recommendations for mediation analyses [14].

Future research could assess how much kinesiphobia affects behavior by using objective measures, such as the actual level of physical activity levels measured through a pedometer or clinical tests such as the six-minute walking test [58]. It might be also 
interesting to investigate the mediating effect of kinesiophobia at different levels of BMI and evaluate its potential role as a moderator.

\section{Conclusions}

The current study improves the current knowledge on the psychological factors that contribute to disability. The findings revealed that in individuals with CLBP and obesity, kinesiophobia mediated the association between pain intensity and disability. Our results highlighted the importance of kinesiophobia as factors that should be evaluated and targeted in rehabilitation interventions to reduce disability in CLBP associated whit obesity.

Author Contributions: G.V. conceived and designed the study protocol and performed the data collections. G.V. carried out literature searches; G.V. designed and carried out the statistical analysis. G.V. drafted the manuscript. G.V., F.S. and E.M.G. interpreted the data; F.S. and E.M.G. collaborated in drafting the manuscript. P.C. supervised the enrollment of participants. R.C., A.G.U. supervised the writing of the manuscript. E.M.G. and G.C. supervised the psychological data collection. All authors critically reviewed and contribute to the final version of the paper. All authors have read and agreed to the published version of the manuscript.

Funding: This research received no external funding.

Institutional Review Board Statement: The study was conducted according to the guidelines of the Declaration of Helsinki of 1975, as revised in 1983, and approved by the Ethics Committee of Istituto Auxologico Italiano (code 2020_02_18_04).

Informed Consent Statement: Informed consent was obtained from all subjects involved in the study.

Data Availability Statement: The data presented in this study are available on request from the corresponding author. The data are not publicly available due to their containing information that could compromise the privacy of research participants.

Conflicts of Interest: The authors declare no conflict of interest.

\section{References}

1. Barke, A.; Schiller, J.; Rief, W.; Treede, R.-D.; Falter, S.; Schäfer, P.; Korwisi, B.; Nugraha, B.; Karst, M.; Gutenbrunner, C. The IASP classification of chronic pain for ICD-11. Pain 2018, 160, 88-94.

2. Hoy, D.; March, L.; Brooks, P.; Blyth, F.; Woolf, A.; Bain, C.; Williams, G.; Smith, E.; Vos, T.; Barendregt, J.; et al. The global burden of low back pain: Estimates from the Global Burden of Disease 2010 study. Ann. Rheum. Dis. 2014, 73, 968-974. [CrossRef]

3. Darnall, B.D.; Ziadni, M.S.; Roy, A.; Kao, M.C.; Sturgeon, J.A.; Cook, K.F.; Lorig, K.; Burns, J.W.; Mackey, S.C. Comparative Efficacy and Mechanisms of a Single-Session Pain Psychology Class in Chronic Low Back Pain: Study Protocol for a Randomized Controlled Trial. Trials 2018, 19, 165. [CrossRef] [PubMed]

4. World Health Organization. World Health Organization Technical Report Series Obesity: Preventing and managing the global epidemic. In Report of a WHO Consultation on Obesity; World Health Organization: Geneva, Switzerland, 2000 ; ISBN 924 120894 5.

5. Heuch, I.; Heuch, I.; Hagen, K.; Zwart, J.A. Body mass index as a risk factor for developing chronic low back pain: A follow-up in the nord-trøndelag health study. Spine (Phila. Pa. 1976) 2013, 38, 133-139. [CrossRef] [PubMed]

6. Heuch, I.; Heuch, I.; Hagen, K.; Zwart, J.A. A comparison of anthropometric measures for assessing the association between body size and risk of chronic low back pain: The HUNT study. PLoS ONE 2015, 10, e0141268. [CrossRef] [PubMed]

7. Janke, E.A.; Collins, A.; Kozak, A.T. Overview of the relationship between pain and obesity: What do we know? Where do we go next? J. Rehabil. Res. Dev. 2007, 44, 245-261. [CrossRef]

8. $\quad$ Freburger, J.K.; Holmes, G.M.; Agans, R.P.; Jackman, A.M.; Darter, J.D.; Wallace, A.S.; Castel, L.D.; Kalsbeek, W.D.; Carey, T.S. The rising prevalence of chronic low back pain. Arch. Intern. Med. 2009, 169, 251-258. [CrossRef]

9. Urquhart, D.M.; Berry, P.; Wluka, A.E.; Strauss, B.J.; Wang, Y.; Proietto, J.; Jones, G.; Dixon, J.B.; Cicuttini, F.M.; Wang, Y.; et al. Increased Fat Mass Is Associated With High Levels of Low Back Pain Intensity and Disability. Spine (Phila. Pa. 1976) 2011, 36, 1320-1325. [CrossRef]

10. Tukker, A.; Visscher, T.L.S.; Picavet, H.S.J. Overweight and health problems of the lower extremities: Osteoarthritis, pain and disability. Public Health Nutr. 2008, 12, 359-368. [CrossRef]

11. Wasser, J.G.; Vasilopoulos, T.; Zdziarski, L.A.; Vincent, H.K. Exercise Benefits for Chronic Low Back Pain in Overweight and Obese Individuals. PM R 2017, 9, 181-192. [CrossRef]

12. Vincent, H.K.; Omli, M.R.; Day, T.; Hodges, M.; Vincent, K.R.; George, S.Z. Fear of Movement, Quality of Life, and Self-Reported Disability in Obese Patients with. Pain Med. (USA) 2011, 35, 154-164. [CrossRef] [PubMed] 
13. Vincent, H.K.; Adams, M.C.B.; Vincent, K.R.; Hurley, R.W. Musculoskeletal pain, fear avoidance behaviors, and functional decline in obesity potential interventions to manage pain and maintain function. Reg. Anesth. Pain Med. 2013, 38, 481-491. [CrossRef] [PubMed]

14. Lee, H.; Hübscher, M.; Moseley, G.L.; Kamper, S.J.; Traeger, A.C.; Mansell, G.; McAuley, J.H. How does pain lead to disability? A systematic review and meta-analysis of mediation studies in people with back and neck pain. Pain 2015, 156, 988-997. [CrossRef]

15. Mühlhauser, Y.; Vogt, L.; Niederer, D. How and how fast does pain lead to disability? A multilevel mediation analysis on structural, temporal and biopsychosocial pathways in patients with chronic nonspecific low back pain. Musculoskelet. Sci. Pract. 2020, 49, 102199. [CrossRef]

16. Marshall, P.W.M.; Schabrun, S.; Knox, M.F. Physical activity and the mediating effect of fear, depression, anxiety, and catastrophizing on pain related disability in people with chronic low back pain. PLoS ONE 2017, 12, e0180788. [CrossRef] [PubMed]

17. Keefe, F.J.; Rumble, M.E.; Scipio, C.D.; Giordano, L.A.; Perri, L.C.M. Psychological aspects of persistent pain: Current state of the science. J. Pain 2004, 5, 195-211. [CrossRef]

18. Giusti, E.M.; Manna, C.; Varallo, G.; Cattivelli, R.; Manzoni, G.M.; Gabrielli, S.; Amario, F.D.; Lacerenza, M.; Castelnuovo, G. The Predictive Role of Executive Functions and Psychological Factors on Chronic Pain after Orthopaedic Surgery: A Longitudinal Cohort Study. Brain Sci. 2020, 10, 685. [CrossRef]

19. Goossens, E.J.B.; Linton, S.J.; Crombez, G.; Leeuw, M.; Boersma, K.; Vlaeyen, J.W.S. The Fear-Avoidance Model of Musculoskeletal Pain: Current State of Scientific Evidence. J. Behav. Med. 2007, 30, 77-94. [CrossRef]

20. Vlaeyen, J.W.S.; Linton, S.J. Fear-avoidance and its consequences in chronic musculoskeletal pain: A state of the art. Pain 2000, 85, 317-332. [CrossRef]

21. Crombez, G.; Eccleston, C.; Van Damme, S.; Vlaeyen, J.W.S.; Karoly, P. Fear-Avoidance Model of Chronic Pain. Clin. J. Pain. 2012, 28, 475-483. [CrossRef]

22. Wideman, T.H.; Asmundson, G.G.; Smeets, R.J.M.; Zautra, A.J.; Simmonds, M.J.; Sullivan, M.J.; Haythornthwaite, J.A.; Edwards, R.R. Rethinking the fear avoidance model: Toward a multidimensional framework of pain-related disability. Pain 2013, 154, 2262-2265. [CrossRef] [PubMed]

23. Vlaeyen, J.W.; Kole-Snijders, A.M.; Boeren, R.G.; van Eek, H. Fear of movement/(re) injury in chronic low back pain and its relation to behavioral performance. Pain 1995, 62, 363-372. [CrossRef]

24. Lethem, J.; Slade, P.D.; Troup, J.D.G.; Bentley, G. Outline of a fear-avoidance model of exaggerated pain perception-I. Behav. Res. Ther. 1983, 21, 401-408. [CrossRef]

25. Celletti, C.; Castori, M.; La Torre, G.; Camerota, F. Evaluation of kinesiophobia and its correlations with pain and fatigue in joint hypermobility syndrome/Ehlers-Danlos syndrome hypermobility type. Biomed Res. Int. 2013, 2013. [CrossRef] [PubMed]

26. Picavet, H.S.J.; Vlaeyen, J.W.S.; Schouten, J.S.A.G. Pain Catastrophizing and Kinesiophobia: Predictors of Chronic Low Back Pain. Am. J. Epidemiol. 2002, 156, 1028-1034. [CrossRef]

27. Luque-suarez, A.; Martinez-calderon, J.; Falla, D. Role of kinesiophobia on pain, disability and quality of life in people suffering from chronic musculoskeletal pain: A systematic review. Br. J. Sports Med. 2018, 53, 554-559. [CrossRef]

28. Vincent, H.K.; Seay, A.N.; Montero, C.; Conrad, B.P.; Hurley, R.W.; Vincent, K.R. Kinesiophobia and fear-avoidance beliefs in overweight older adults with chronic low-back pain: Relationship to walking endurance-Part II. Am. J. Phys. Med. Rehabil. 2013, 92, 439-445. [CrossRef]

29. Varallo, G.; Giusti, E.M.; Scarpina, F.; Cattivelli, R.; Capodaglio, P.; Castelnuovo, G. The Association of Kinesiophobia and Pain Catastrophizing with Pain-Related Disability and Pain Intensity in Obesity and Chronic Lower-Back Pain. Brain Sci. 2020, 11, 11. [CrossRef]

30. Vincent, H.K.; Lamb, K.M.; Day, T.I.; Tillman, S.M.; Vincent, K.R.; George, S.Z. Morbid Obesity Is Associated With Fear of Movement and Lower Quality of Life in Patients With Knee Pain-Related Diagnoses. PMRJ 2010, 2, 713-722. [CrossRef]

31. Vincent, H.K.; Vincent, K.R.; Seay, A.N.; Hurley, R.W. Functional impairment in obesity: A focus on knee and back pain. Pain Manag. 2011, 1, 427-439. [CrossRef]

32. Costa, L.D.C.M.; Maher, C.G.; McAuley, J.H.; Hancock, M.J.; Smeets, R.J.E.M. Self-efficacy is more important than fear of movement in mediating the relationship between pain and disability in chronic low back pain. Eur. J. Pain 2011, 15, $213-219$. [CrossRef] [PubMed]

33. Kamper, S.J.; Maher, C.G.; Menezes Costa, L.D.C.; McAuley, J.H.; Hush, J.M.; Sterling, M. Does fear of movement mediate the relationship between pain intensity and disability in patients following whiplash injury? A prospective longitudinal study. Pain 2012, 153, 113-119. [CrossRef] [PubMed]

34. Padua, L.; Ceccarelli, E.; Romanini, E.; Zanoli, G.; Bondì, R. Italian version of the Roland Disability Questionnaire, specific for low back pain: Cross-cultural adaptation and validation. Eur. Spine J. 2002, 11, 126-129. [CrossRef] [PubMed]

35. Roland, M.O. The natural history of back pain. Practitioner 1983, 227, 1119-1122.

36. Ritter, P.L.; González, V.M.; Laurent, D.D.; Lorig, K.R. Measurement of pain using the visual numeric scale. J. Rheumatol. 2006, 33, 574-580.

37. Breivik, H.; Borchgrevink, P.C.; Allen, S.M.; Rosseland, L.A.; Romundstad, L.; Hals, E.K.B.; Kvarstein, G.; Stubhaug, A. Assessment of pain. Br. J. Anaesth. 2008, 101, 17-24. [CrossRef] 
38. Monticone, M.; Giorgi, I.; Baiardi, P.; Barbieri, M.; Rocca, B.; Bonezzi, C. Development of the Italian Version of the Tampa Scale of Kinesiophobia (TSK-I): Cross-Cultural Adaptation, Factor Analysis, Reliability, and Validity. Spine (Phila. Pa. 1976) 2010, 35, 1241-1246. [CrossRef]

39. Woby, S.R.; Roach, N.K.; Urmston, M.; Watson, P.J. Psychometric properties of the TSK-11: A shortened version of the Tampa Scale for Kinesiophobia. Pain 2005, 117, 137-144. [CrossRef]

40. Cohen, J. Set Correlation and Contingency Tables. Appl. Psychol. Meas. 1988, 12, 425-434. [CrossRef]

41. MacKinnon, D.P. Introduction to Statistical Mediation Analysis; Lawrence Erlbaum Associates: New York, NY, USA, 2008.

42. Baron, R.M.; Kenny, D.A. The moderator-mediator variable distinction in social psychological research: Conceptual, strategic, and statistical considerations. J. Pers. Soc. Psychol. 1986, 51, 1173-1182. [CrossRef]

43. Jamovi (version 1.2). The Jamovi Project 2020. Available online: https://www.jamovi.org/about.html (accessed on 21 May 2021).

44. Preacher, K.J.; Hayes, A.F. Asymptotic and resampling strategies for assessing and comparing indirect effects in multiple mediator models. Behav. Res. Methods 2008, 40, 879-891. [CrossRef] [PubMed]

45. Hayes, A.F. Introduction to Mediation, Moderation and Conditional Process Analysis; Guiford Press: New York, NY, USA, 2013.

46. Fritz, M.S.; MacKinnon, D.P. Required sample size to detect the mediated effect. Psychol. Sci. 2007, 18, 233-239. [CrossRef]

47. Tabachnick, B.G.; Fidell, L.S.; Ullman, J.B. Using Multivariate Statistics; Pearsons: Boston, MA, USA, 2007.

48. Gheldof, E.L.M.; Vinck, J.; Van den Bussche, E.; Vlaeyen, J.W.S.; Hidding, A.; Crombez, G. Pain and pain-related fear are associated with functional and social disability in an occupational setting: Evidence of mediation by pain-related fear. Eur. J. Pain 2006, 10, 513. [CrossRef] [PubMed]

49. Ballantyne, J.C.; Sullivan, M.D. Intensity of Chronic Pain-The Wrong Metric? N. Engl. J. Med. 2015, 373, 2098-2099. [CrossRef]

50. Sullivan, M.D.; Ballantyne, J.C. Must we reduce pain intensity to treat chronic pain? Pain 2016, 157, 65-69. [CrossRef] [PubMed]

51. Lee, H.; Mansell, G.; McAuley, J.H.; Kamper, S.J.; Hübscher, M.; Moseley, G.L.; Wolfenden, L.; Hodder, R.K.; Williams, C.M. Causal mechanisms in the clinical course and treatment of back pain. Best Pract. Res. Clin. Rheumatol. 2016, 30, 1074-1083. [CrossRef] [PubMed]

52. GK, F. Therapeutic exercise for knee osteoarthritis: Considering factors that may influence outcome. Eur. Medicophys. 2005, 41, 163-171.

53. Brooks, C.; Siegler, J.C.; Cheema, B.S.; Marshall, P.W.M. No relationship between body mass index and changes in pain and disability after exercise rehabilitation for patients with mild to moderate chronic low back pain. Spine (Phila. Pa. 1976) 2013, 38, 2190-2195. [CrossRef]

54. Leboeuf-Yde, C. Body Weight and Low Back Pain. Spine (Phila. Pa. 1976) 2000, 25, 226. [CrossRef] [PubMed]

55. Han, T.S.; Schouten, J.S.A.G.; Lean, M.E.J.; Seidell, J.C. The prevalence of low back pain and associations with body fatness, fat distribution and height. Int. J. Obes. 1997, 21, 600-607. [CrossRef]

56. Donnelly, J.E.; Smith, B.; Jacobsen, D.J.; Kirk, E.; DuBose, K.; Hyder, M.; Bailey, B.; Washburn, R. The role of exercise for weight loss and maintenance. Best Pract. Res. Clin. Gastroenterol. 2004, 18, 1009-1029. [CrossRef]

57. Egger, G.; Dixon, J. Non-nutrient causes of low-grade, systemic inflammation: Support for a "canary in the mineshaft" view of obesity in chronic disease. Obes. Rev. 2011, 12, 339-345. [CrossRef] [PubMed]

58. American Thoracic Society American Thoracic Society ATS Statement: Guidelines for the Six-Minute Walk Test. Am. J. Respir. Crit. Care Med. 2002, 166, 111-117. [CrossRef] [PubMed] 